\title{
Farey fractions and sums over coprime pairs
}

\author{
by \\ MASAYOSHI Hata (Kyoto)
}

1. In 1964, H. Gupta [2] showed the equality

$$
\sum_{b=1}^{\infty} \sum_{\substack{1 \leq d \leq b \\(b, d)=1}} \frac{1}{b^{2}(b+d)}=\frac{3}{4}
$$

by using the well-known properties of the Farey series (see, for example, G. H. Hardy and E. M. Wright [3, Chapter III]). Later J. Lehner and M. Newman [5] obtained a formula which reduces a sum involving Farey fractions to one which does not.

The aim of this note is to give another method to evaluate this kind of infinite sums by applying the construction of Farey fractions to Schauder expansions for continuous functions. For example, we will show that

and

$$
\begin{gathered}
\sum_{b=1}^{\infty} \sum_{\substack{1 \leq d \leq b \\
(b, d)=1}} \frac{1}{b d(b+d)}=\frac{5}{4}, \\
\sum_{b=1}^{\infty} \sum_{\substack{1 \leq d \leq b \\
(b, d)=1}} \frac{1}{b d(b+d)^{2}}=\frac{3}{8}
\end{gathered}
$$

$$
\sum_{b=1}^{\infty} \sum_{\substack{1 \leq d \leq b \\(b, d)=1}} \frac{1}{(b d(b+d))^{2}}=\frac{7}{24} .
$$

Moreover, it will be shown that Euler's constant

$$
\gamma=\lim _{n \rightarrow \infty}\left(1+\frac{1}{2}+\ldots+\frac{1}{n}-\log n\right)=0.5772156649 \ldots
$$

can also be expressed by a similar sum (Theorem 4.1), which is probably a new formula for $\gamma$ as far as the author knows. It is not known whether $\gamma$ is rational or irrational. (For the history of $\gamma$, see J. W. L. Glaisher [1].) 
2. Let $G_{1}=\{0 / 1,1 / 1\}$ and define $G_{n+1}$ inductively by the union of $G_{n}$ and all their mediants, arranged in ascending order. For example, $G_{4}$ consists of

$$
\left\{\frac{0}{1}, \frac{1}{4}, \frac{1}{3}, \frac{2}{5}, \frac{1}{2}, \frac{3}{5}, \frac{2}{3}, \frac{3}{4}, \frac{1}{1}\right\} .
$$

We call $G_{n}$ a modified Farey series of order $n$. The series $G_{n}$ can also be regarded naturally as a finite subset of $[0,1]$. Since the greatest denominator of the terms in $G_{n}$ is the $(n+1)$ th term of Fibonacci's series $\left\{u_{n}\right\}$, we have $F_{n} \subset G_{n} \subset F_{u_{n+1}}$, where $F_{n}$ is the usual Farey series of order $n$. Let $\mathcal{F}_{n}$ be the collection of intervals $I=[a / b, c / d]$, where $a / b$ and $c / d$ run over all successive terms of $G_{n}$. We call $\mathcal{F}_{n}$ the collection of fundamental intervals of order $n$. Note that

$$
\sum_{I \in \mathcal{F}_{n}} \frac{1}{b d}=\sum_{I \in \mathcal{F}_{n}}|I|=1
$$

where $|I|$ denotes the length of $I$ and that $b+d \geq n+1$ since $F_{n} \subset G_{n}$. It is known that, for any relatively prime positive integers $b$ and $d$, there exists a unique fundamental interval $I$ of the form $[a / b, c / d]\left({ }^{1}\right)$. Put $\mathcal{F}=\bigcup_{n=1}^{\infty} \mathcal{F}_{n}$ for brevity. The collection of open intervals $\{\operatorname{int} I\}_{I \in \mathcal{F}}$ forms a net, that is, any two such intervals are either disjoint or else one is contained in the other.

3. We now define our Schauder expansions associated with the modified Farey series $\left\{G_{n}\right\}$. For a different type of Schauder bases associated with dyadic rationals, see M. Hata and M. Yamaguti [4]. For any fundamental interval $I=[a / b, c / d]$, define a piecewise linear function $S_{I}(x)$ by

$$
S_{I}(x)=\frac{b+d}{2}(|a-b x|+|c-d x|-|a+c-(b+d) x|),
$$

which we call a Schauder base. Note that $\operatorname{supp}\left(S_{I}\right)=I$ and $\left\|S_{I}\right\|=1$, where $\|f\|$ denotes the usual uniform norm for $f \in C[0,1]$. It is easily seen that the system $\left\{S_{I}^{\prime}(x)\right\}_{I \in \mathcal{F}}$ satisfies the orthogonality property:

$$
\int_{0}^{1} S_{I}^{\prime}(x) S_{J}^{\prime}(x) d x= \begin{cases}(b+d)^{2} & \text { if } I=J=[a / b, c / d], \\ 0 & \text { otherwise. }\end{cases}
$$

Define also a linear functional $c_{I}(f)$ on $C[0,1]$ by

$$
c_{I}(f)=f\left(\frac{a+c}{b+d}\right)-\frac{b}{b+d} f\left(\frac{a}{b}\right)-\frac{d}{b+d} f\left(\frac{c}{d}\right) .
$$

It is obvious that $c_{I}(f+g)=c_{I}(f)$ whenever $g(x)$ is linear on $I$. Then we have

$\left({ }^{1}\right)$ Throughout this paper we always assume that the small letters $a$ and $c$ are the unique integral solutions of $b c-a d=1$ satisfying $0 \leq a<b$ and $1 \leq c \leq d$. 
Lemma 3.1. Every $f \in C[0,1]$ can be uniquely expanded in Schauder bases as follows:

$$
f(x)=f(0)+\Delta_{[0,1]}(f) x+\sum_{n=1}^{\infty} \sum_{I \in \mathcal{F}_{n}} c_{I}(f) S_{I}(x),
$$

where $\Delta_{[0,1]}(f)=f(1)-f(0)$. This series converges uniformly in $[0,1]$.

Proof. Let

$$
f_{n}(x)=f(0)+\Delta_{[0,1]}(f) x+\sum_{i=1}^{n} \sum_{I \in \mathcal{F}_{i}} c_{I}(f) S_{I}(x)
$$

for $n \geq 1$. It is easily seen that $f(x)=f_{n}(x)$ for every $x \in G_{n+1}$. By the uniform continuity of $f(x)$, for any $\varepsilon>0$ there exists $\delta>0$ satisfying $|f(x)-f(y)|<\varepsilon$ whenever $|x-y|<\delta$. Take $n$ to be so large that $|I|<\delta$ for every $I \in \mathcal{F}_{n+1}$. Then, for any $x \in[0,1]$ there exists a fundamental interval $I=[a / b, c / d]$ of order $n+1$ satisfying $x \in I$; hence

$\left|f(x)-f_{n}(x)\right| \leq\left|f(x)-f_{n}\left(\frac{a}{b}\right)\right|+\left|f_{n}\left(\frac{a}{b}\right)-f_{n}(x)\right|<\varepsilon+\left|f_{n}\left(\frac{a}{b}\right)-f_{n}(x)\right|$.

Since $f_{n}(x)$ is linear on $I$, we get

$$
\left|f_{n}\left(\frac{a}{b}\right)-f_{n}(x)\right| \leq\left|f_{n}\left(\frac{a}{b}\right)-f_{n}\left(\frac{c}{d}\right)\right|=\left|f\left(\frac{a}{b}\right)-f\left(\frac{c}{d}\right)\right|<\varepsilon .
$$

Therefore $\left\|f-f_{n}\right\|<2 \varepsilon$, which completes the proof.

The next lemma will be useful for calculation of the coefficient $c_{I}(f)$. For any $r \in \mathbb{N}$ we denote by $C^{r}[0,1]$ the set of all $r$ times continuously differentiable functions defined on some open neighborhood of $[0,1]$.

Lemma 3.2. For any $g \in C^{2}[0,1]$ and any $I=[a / b, c / d] \in \mathcal{F}$ we have

$$
c_{I}(g)=-\frac{1}{(b+d)^{2}} \int_{I} S_{I}(x) g^{\prime \prime}(x) d x .
$$

The proof is easily supplied by partial integration. Thus, multiplying both sides of (3.1) by $g^{\prime \prime}(x)$ and integrating term-by-term, we get, using Lemma 3.2,

TheOrem 3.3. For any $f \in C[0,1]$ and $g \in C^{2}[0,1]$ we have

$$
\begin{aligned}
\sum_{n=1}^{\infty} \sum_{I \in \mathcal{F}_{n}}(b+d)^{2} c_{I}(f) c_{I}(g) & \\
= & \Delta_{[0,1]}\left(f g^{\prime}\right)-\Delta_{[0,1]}(f) \Delta_{[0,1]}(g)-\int_{0}^{1} f(x) g^{\prime \prime}(x) d x .
\end{aligned}
$$

As a corollary, we have immediately 
Corollary 3.4. For any $f \in C^{2}[0,1]$,

$$
\int_{0}^{1}\left(f^{\prime}(x)\right)^{2} d x=\left(\Delta_{[0,1]}(f)\right)^{2}+\sum_{I \in \mathcal{F}}(b+d)^{2}\left(c_{I}(f)\right)^{2} .
$$

Perhaps one would think that this is an analogue of Parseval's formula in Fourier analysis. Putting $g(x)=x^{2}$, we get $c_{I}(g)=-1 /\left(b d(b+d)^{2}\right)$ from Lemma 3.2. In this case the formula in Theorem 3.3 takes the following simpler form.

Corollary 3.5. For any $f \in C[0,1]$ we have

$$
\sum_{n=1}^{\infty} \sum_{I \in \mathcal{F}_{n}} \frac{c_{I}(f)}{b d}=2 \int_{0}^{1} f(x) d x-f(0)-f(1) .
$$

4. We give several examples of the summation formulae in the previous section, in which the series converge absolutely. As the first example, we take $f(x)=x^{2}$ in Corollary 3.5; hence we get

$$
\sum_{(b, d)=1} \frac{1}{(b d(b+d))^{2}}=\frac{1}{3},
$$

from which the equality (1.4) follows easily.

As the second example, we take $f(x)=y /(1+x y)$ and $g(x)=z /(1+x z)$ in Theorem 3.3, where $y, z>-1$ are real parameters. Then it follows that

$$
\begin{array}{r}
\sum_{(b, d)=1} \frac{1}{(a y+b)(c y+d)((a+c) y+b+d)(a z+b)(c z+d)((a+c) z+b+d)} \\
= \begin{cases}\frac{1}{(y-z)^{3}}\left(\frac{1+y}{1+z}-\frac{1+z}{1+y}-2 \log \frac{1+y}{1+z}\right) & \text { if } y \neq z, \\
\frac{1}{3(1+y)^{3}} & \text { if } y=z .\end{cases}
\end{array}
$$

For example, putting $y=1, z=0$ and $y=z=1$, we get

$$
\sum_{(b, d)=1} \frac{1}{b d(b+d)(a+b)(c+d)(a+b+c+d)}=\frac{3}{2}-2 \log 2
$$

and

$$
\sum_{(b, d)=1} \frac{1}{((a+b)(c+d)(a+b+c+d))^{2}}=\frac{1}{24}
$$

respectively.

As the third example, we take

$$
\psi(x)=x\left\{\frac{1}{x}\right\}\left(1-\left\{\frac{1}{x}\right\}\right),
$$


where $\{x\}$ denotes the fractional part of $x$. Then clearly $c_{I}(\psi)=0$ if $I \in \mathcal{F}^{*}=\{[0 / 1,1 / n]: n \in \mathbb{N}\}$ and $c_{I}(\psi)=1 /(a c(a+c)(b+d))$ otherwise. Since

$$
\begin{aligned}
\int_{0}^{1} \psi(x) d x & =\int_{0}^{1} x(1-x) \sum_{n=1}^{\infty} \frac{1}{(n+x)^{3}} d x \\
& =\lim _{N \rightarrow \infty}\left(1+\frac{1}{2}+\ldots+\frac{1}{N}-\frac{N+1}{2 N}-\log N\right)=\gamma-\frac{1}{2},
\end{aligned}
$$

we thus have from Corollary 3.5

TheOREM 4.1. Euler's constant $\gamma$, defined in (1.5), can be expressed as follows:

$$
\gamma=\frac{1}{2}+\frac{1}{2} \sum_{\substack{(b, d)=1 \\ b \geq 2}} \frac{1}{a b c d(a+c)(b+d)} .
$$

Note that the mapping $\tau: \mathcal{F} \backslash \mathcal{F}^{*} \rightarrow 4 \mathbb{N}$ defined by $\tau([a / b, c / d])=$ $a b c d(a+c)(b+d)$ is not injective. For example, $\tau([2 / 7,1 / 3])=\tau([5 / 6,1 / 1])$ and $\tau([2 / 3,3 / 4])=\tau([1 / 2,4 / 7])$, etc. Probably one will find infinitely many such pairs of fundamental intervals.

Similarly, taking

$$
f(x)=\frac{x \psi(x)}{1+1 / x-\{1 / x\}} \quad \text { and } \quad f(x)=x \psi(x)
$$

in Corollary 3.5, we get

$$
\sum_{(b, d)=1} \frac{[d / c]}{(b d(b+d))^{2}}=\frac{1}{3} \zeta(3)
$$

and

$$
\sum_{(b, d)=1} \frac{[d / c]+[d / c]^{2}}{(b d(b+d))^{2}}=\frac{2}{3} \zeta(2)
$$

respectively, where $\zeta(z)$ is the Riemann zeta function. (Note that the lefthand sides of (4.4) and (4.5) include the terms corresponding to $\mathcal{F}^{*}$.)

5. To obtain another summation formula we need a few lemmas. For any $I=[a / b, c / d] \in \mathcal{F}$ we put $l(I)=a / b, r(I)=c / d$ and $m(I)=(a+c) /(b+d)$ for brevity. For any Farey fraction $\sigma \in G \equiv \bigcup_{n>1} G_{n}$, we denote by $\mathcal{G}_{\sigma}$ the collection of all fundamental intervals $I$ satisfying either $l(I)=\sigma$ or $r(I)=\sigma$. For example, $\mathcal{G}_{1 / 2}$ consists of all intervals of the form $\left[\frac{n-1}{2 n-1}, \frac{1}{2}\right]$ and $\left[\frac{1}{2}, \frac{n}{2 n-1}\right]$ for $n \in \mathbb{N}$.

Then clearly each fundamental interval $I$ belongs to precisely two collections $\mathcal{G}_{l(I)}$ and $\mathcal{G}_{r(I)}$. In other words, we have 
Lemma 5.1. Let $\nu$ be a function on $\mathcal{F}$ such that $\sum_{I \in \mathcal{F}} \nu(I)$ converges absolutely. Then

$$
2 \sum_{I \in \mathcal{F}} \nu(I)=\sum_{\sigma \in G} \sum_{J \in \mathcal{G}_{\sigma}} \nu(J) .
$$

Let $\omega: \mathcal{F} \rightarrow \mathbb{R}$ be a weight function defined by $\omega([a / b, c / d])=1 /(b+d)$. Then we have

Lemma 5.2. For any $I \in \mathcal{F}$,

$$
\omega(I) S_{I}(x)=\sum_{J \in \mathcal{G}_{m(I)}} \omega(J) S_{J}(x),
$$

where the right-hand side converges uniformly on any compact subset in $[0,1] \backslash\{m(I)\}$.

Proof. Obviously $I$ contains all $J \in \mathcal{G}_{m(I)}$ and any $J \in \mathcal{G}_{m(I)}$ contained in $I^{-}=[l(I), m(I)]$ takes the form

$$
J_{n} \equiv\left[\frac{n a+(n-1) c}{n b+(n-1) d}, \frac{a+c}{b+d}\right], \quad n \in \mathbb{N} .
$$

Put $\xi_{n}(x)=\omega(I) S_{I}(x)-\sum_{i=1}^{n} \omega\left(J_{i}\right) S_{J_{i}}(x)$ for all $n \geq 1$. Then it suffices to show that

$$
\xi_{n}(x)= \begin{cases}0 & \text { for } l(I) \leq x \leq l\left(J_{n+1}\right), \\ \frac{1}{\omega\left(J_{n}\right)}\left(x-l\left(J_{n+1}\right)\right) & \text { for } l\left(J_{n+1}\right) \leq x \leq m(I),\end{cases}
$$

by induction on $n$, since $S_{J_{i}}(x) \equiv 0$ on $\left[l(I), l\left(J_{n+1}\right)\right]$ for all $i \geq n+1$.

Clearly (5.2) holds for $n=1$, since $\omega(I) S_{I}(x)=b x-a$ on $[l(I), m(I)]$. Suppose now that (5.2) holds for $n=k \geq 1$. Since

$$
\frac{1}{\omega\left(J_{k}\right)}\left(l\left(J_{k+2}\right)-l\left(J_{k+1}\right)\right)=\frac{1}{(k+2) b+(k+1) d}=\omega\left(J_{k+1}\right),
$$

it follows that $\xi_{k+1}(x) \equiv 0$ on $\left[l(I), l\left(J_{k+2}\right)\right]$ and that $\xi_{k+1}$ is a linear function on $\left[l\left(J_{k+2}\right), m(I)\right]$ through the two points $\left(l\left(J_{k+2}\right), 0\right)$ and $(m(I), \omega(I))$. Hence (5.2) also holds for $n=k+1$, since $m(I)-l\left(J_{k+2}\right)=\omega(I) \omega\left(J_{k+1}\right)$.

The similar argument can be applied to the case $I^{+}=[m(I), r(I)]$. This completes the proof.

Multiplying both sides of (5.1) by $f^{\prime \prime}(x)$ and applying Lemma 3.2, we get

$$
\nu(I)=\sum_{J \in \mathcal{G}_{m(I)}} \nu(J)
$$

where $\nu(I)=c_{I}(f) / \omega(I)$. Note that the series $\sum_{n \geq 1} \sum_{I \in \mathcal{F}_{n}} \nu(I)$ converges 
absolutely for any $f \in C^{2}[0,1]$, since it follows from Lemma 3.2 that

$$
\begin{aligned}
\sum_{I \in \mathcal{F}}(b+d)^{2-\varepsilon}\left|c_{I}(f)\right| & \leq\left\|f^{\prime \prime}\right\| \sum_{(b, d)=1} \frac{1}{b d(b+d)^{\varepsilon}} \\
& \leq\left\|f^{\prime \prime}\right\| \sum_{m, n=1}^{\infty} \frac{1}{m n(m+n)^{\varepsilon}}<\infty
\end{aligned}
$$

for any $\varepsilon>0$. Hence, from Lemma 5.1,

$$
2 \sum_{I \in \mathcal{F}} \nu(I)=\sum_{J \in \mathcal{G}_{0} / 1} \nu(J)+\sum_{J \in \mathcal{G}_{1 / 1}} \nu(J)+\sum_{I \in \mathcal{F}} \nu(I),
$$

since for every Farey fraction $\sigma \in G$ except for $0 / 1$ and $1 / 1$ there exists a unique fundamental interval $I$ satisfying $m(I)=\sigma$. We also have

$$
\begin{aligned}
\sum_{J \in \mathcal{G}_{0 / 1}} \nu(J)+\sum_{J \in \mathcal{G}_{1 / 1}} \nu(J) & =\sum_{n=1}^{\infty}(n+1)\left(c_{[0 / 1,1 / n]}(f)+c_{[(n-1) / n, 1 / 1]}(f)\right) \\
& =\sum_{n=1}^{\infty}\left(A_{n+1}-A_{n}\right)
\end{aligned}
$$

where $A_{n}=n\left(\Delta_{[0 / 1,1 / n]}(f)-\Delta_{[(n-1) / n, 1 / 1]}(f)\right), n \in \mathbb{N}$. Since $A_{n} \rightarrow f^{\prime}(0)-$ $f^{\prime}(1)$ as $n \rightarrow \infty$, we thus proved

Theorem 5.3. For any $f \in C^{2}[0,1]$ we have

$$
\sum_{n=1}^{\infty} \sum_{I \in \mathcal{F}_{n}}(b+d) c_{I}(f)=-\Delta_{[0,1]}\left(f^{\prime}\right) .
$$

For example, taking $f(x)=x^{2}$ in Theorem 5.3 , we get

$$
\sum_{(b, d)=1} \frac{1}{b d(b+d)}=2,
$$

from which the equality (1.2) follows easily. In particular, combining (1.1) and (1.2), we obtain

$$
\sum_{b=1}^{\infty} \sum_{\substack{1 \leq d \leq b \\(b, d)=1}} \frac{1}{b^{2} d}=2 .
$$

As another example, we take $f(x)=y /(1+x y)$ in Theorem 5.3. Then

$$
\sum_{(b, d)=1} \frac{1}{(a y+b)(c y+d)((a+c) y+b+d)}=\frac{2+y}{(1+y)^{2}}
$$


for any $y>-1$. In particular, putting $y=1$, we get

$$
\sum_{(b, d)=1} \frac{1}{(a+b)(c+d)(a+b+c+d)}=\frac{3}{4} .
$$

Compare with (4.3).

6. Let $\left\{\mathcal{U}_{n}\right\}_{n \geq 1}$ be any sequence of collections of finite fundamental intervals. For any function $\nu: \mathcal{F} \rightarrow \mathbb{R}$ and $n \geq 1$, we put

$$
S_{n}=\sum_{I \in \mathcal{U}_{n}} \nu(I)
$$

Then we have immediately

$$
S_{1}-S_{N+1}=\sum_{n=1}^{N}\left(S_{n}-S_{n+1}\right)=\sum_{n=1}^{N}\left(\sum_{I \in \mathcal{U}_{n} \backslash \mathcal{U}_{n+1}} \nu(I)-\sum_{I \in \mathcal{U}_{n+1} \backslash \mathcal{U}_{n}} \nu(I)\right)
$$

for all $N \geq 1$. Indeed, let $\mathcal{E}_{n}$ be the collection of intervals $I=[a / b, c / d]$, where $a / b$ and $c / d$ run over all successive terms of the usual Farey series $F_{n}$. Then one has Lehner and Newman's formula by applying the above simple principle to the sequence $\left\{\mathcal{E}_{n}\right\}_{n \geq 1}$.

For our series $\left\{\mathcal{F}_{n}\right\}_{n \geq 1}$, we have clearly $\mathcal{F}_{i} \cap \mathcal{F}_{j}=\emptyset$ for any $i \neq j$ and

$$
S_{n}-S_{n+1}=\sum_{I \in \mathcal{F}_{n}}\left(\nu(I)-\nu\left(I^{+}\right)-\nu\left(I^{-}\right)\right),
$$

where $I^{+}=[m(I), r(I)]$ and $I^{-}=[l(I), m(I)]$. Thus we have

TheOREM 6.1. Let $\nu$ be a function on $\mathcal{F}$ such that $\sum_{I \in \mathcal{F}_{n}} \nu(I) \rightarrow 0$ as $n \rightarrow \infty$. Then

$$
\sum_{n=1}^{\infty} \sum_{I \in \mathcal{F}_{n}}\left(\nu(I)-\nu\left(I^{+}\right)-\nu\left(I^{-}\right)\right)=\nu([0,1]) .
$$

For example, taking $\nu(I)=1 /(b d)^{2}$ in Theorem 6.1 , we get

$$
\sum_{(b, d)=1} \frac{1}{b d(b+d)^{2}}=\frac{1}{2}
$$

from which the equality (1.3) follows easily. We get (4.1) again if we take $\nu(I)=1 /(b d)^{3}$.

As another example, taking $\nu(I)=1 /(b d(b+d))$ in Theorem 6.1 , we get

$$
\sum_{(b, d)=1} \frac{1}{(b+d)(b+2 d)(2 b+d)}=\frac{1}{6} \text {. }
$$


Note that Gupta's equality (1.1) comes now from (6.2) and Theorem 6.1 if one takes

$$
\nu(I)=\frac{1}{(b+d) \max \left\{b^{2}, d^{2}\right\}},
$$

since $\nu\left(I^{+}\right)+\nu\left(I^{-}\right)=3 /((b+d)(b+2 d)(2 b+d))$. Similarly, taking

$$
\nu(I)=\frac{1}{b d \max \left\{b^{2}, d^{2}\right\}},
$$

we get from (6.1),

$$
\sum_{b=1}^{\infty} \sum_{\substack{1 \leq d \leq b \\(b, d)=1}} \frac{1}{b^{3} d}=\frac{5}{4} .
$$

Compare with (5.4).

7. Finally, we remark that

$$
\sum_{I \in \mathcal{F}} \omega(I) S_{I}(x)= \begin{cases}1-1 / q & \text { if } x=p / q \in \mathbb{Q} \cap[0,1] \text { with }(p, q)=1 \\ 1 & \text { if } x \in[0,1] \backslash \mathbb{Q} .\end{cases}
$$

This is a typical example in elementary analysis, a function which is continuous at every irrational point in $(0,1)$ but discontinuous at every rational point in $[0,1]$. For any rational $x \in[0,1],(7.1)$ is easily shown by induction on the order of modified Farey series. Moreover, this implies that

$$
\sum_{n=1}^{N} \sum_{I \in \mathcal{F}_{n}} \omega(I) S_{I}(x)<1
$$

for any $N \geq 1$, since the left-hand side is a linear function on each fundamental interval $I \in \mathcal{F}_{N+1}$. Thus the series $\sum_{I \in \mathcal{F}} \omega(I) S_{I}(\varrho)$ converges absolutely for each fixed irrational $\varrho \in(0,1)$. Thus, putting $\nu(I)=\omega(I) S_{I}(\varrho)$, we have

$$
\nu(I)=\sum_{J \in \mathcal{G}_{m(I)}} \nu(J)
$$

from Lemma 5.2, and the same argument as in the proof of Theorem 5.3 implies (7.1), as required. In particular, there exist no continuous functions $f$ satisfying $c_{I}(f)=1 /(b+d)$ for any $I \in \mathcal{F}$.

On the other hand, if we assume that $\sum_{I \in \mathcal{F}}\left|c_{I}(f)\right|<\infty$ for $f \in C[0,1]$, then $f(x)$ must be absolutely continuous. To see this, we need a simple lemma.

For any $I \in \mathcal{F}$ let $\mathcal{H}_{I}$ be the collection of all fundamental intervals $J$ which contain $I$ as a proper subset. For example, $\mathcal{H}_{[0,1]}=\emptyset$ and $\mathcal{H}_{[1 / 3,1 / 2]}$ consists of the two intervals $[0 / 1,1 / 1]$ and $[0 / 1,1 / 2]$. Put $\Delta_{I}(f)=f(r(I))-$ $f(l(I))$ for all $I \in \mathcal{F}$. Then 
Lemma 7.1. For any $f \in C[0,1]$ and any $I \in \mathcal{F}$,

$$
\Delta_{I}(f)=|I| \Delta_{[0,1]}(f)+\sum_{J \in \mathcal{H}_{I}} c_{J}(f) \int_{I} S_{J}^{\prime}(x) d x .
$$

Proof. We prove this by induction on the order of fundamental intervals. Clearly $(7.2)$ holds for $I=[0,1] \in \mathcal{F}_{1}$. Suppose now that $(7.2)$ holds for every fundamental interval $I$ of order less than or equal to $k$ for some $k \geq 1$. Take any $I \in \mathcal{F}_{k+1}$ and $K \in \mathcal{F}_{k}$ such that $I$ is a proper subset of $K$. Then $\mathcal{H}_{I}=\{K\} \cup \mathcal{H}_{K}$ and

$$
\begin{aligned}
\sum_{J \in \mathcal{H}_{K}} c_{J}(f) \int_{I} S_{J}^{\prime}(x) d x & =\frac{|I|}{|K|} \sum_{J \in \mathcal{H}_{K}} c_{J}(f) \int_{K} S_{J}^{\prime}(x) d x \\
& =\frac{|I|}{|K|} \Delta_{K}(f)-|I| \Delta_{[0,1]}(f),
\end{aligned}
$$

since $S_{J}^{\prime}(x)$ is constant on $K$ for every $J \in \mathcal{H}_{K}$. Hence

$$
|I| \Delta_{[0,1]}(f)+\sum_{J \in \mathcal{H}_{I}} c_{J}(f) \int_{I} S_{J}^{\prime}(x) d x=\frac{|I|}{|K|} \Delta_{K}(f)+c_{K}(f) \int_{I} S_{K}^{\prime}(x) d x .
$$

First we suppose that $l(I)=l(K)$; that is, $r(I)=m(K)$. We then have $\int_{I} S_{K}^{\prime}(x) d x=1$ and

$$
\frac{|I|}{|K|} \Delta_{K}(f)+c_{K}(f)=f(m(K))-f(l(K))=\Delta_{I}(f) .
$$

Similarly we get the same conclusion in the case $r(I)=r(K)$. Therefore (7.2) holds for every $I \in \mathcal{F}_{k+1}$ and this completes the proof.

Theorem 7.2. Suppose that $\sum_{I \in \mathcal{F}}\left|c_{I}(f)\right|<\infty$ for $f \in C[0,1]$. Then $f(x)$ must be absolutely continuous and

$$
f^{\prime}(x)=\Delta_{[0,1]}(f)+\sum_{n=1}^{\infty} \sum_{I \in \mathcal{F}_{n}} c_{I}(f) S_{I}^{\prime}(x)
$$

holds almost everywhere.

Proof. Put

$$
\varphi(x)=\Delta_{[0,1]}(f)+\sum_{n=1}^{\infty} \sum_{I \in \mathcal{F}_{n}} c_{I}(f) S_{I}^{\prime}(x) .
$$

Then $\varphi(x)$ is an integrable function on $[0,1]$, since

$$
\sum_{n=1}^{\infty} \sum_{I \in \mathcal{F}_{n}}\left|c_{I}(f)\right| \int_{0}^{1}\left|S_{I}^{\prime}(x)\right| d x=2 \sum_{I \in \mathcal{F}}\left|c_{I}(f)\right|<\infty .
$$


Integrating term-by-term and using Lemma 7.1, we have

$$
\begin{aligned}
\int_{I} \varphi(x) d x & =|I| \Delta_{[0,1]}(f)+\sum_{n=1}^{\infty} \sum_{J \in \mathcal{F}_{n}} c_{J}(f) \int_{I} S_{J}^{\prime}(x) d x \\
& =|I| \Delta_{[0,1]}(f)+\sum_{J \in \mathcal{H}_{I}} c_{J}(f) \int_{I} S_{J}^{\prime}(x) d x=\Delta_{I}(f)
\end{aligned}
$$

for any $I \in \mathcal{F}$, since $\int_{I} S_{J}^{\prime}(x) d x=0$ if either $J \subset I$ or int $J \cap \operatorname{int} I=\emptyset$ holds. Thus

$$
\int_{0}^{r(I)} \varphi(x) d x=f(r(I))-f(0)
$$

for any $I \in \mathcal{F}$. Since $\{r(I): I \in \mathcal{F}\}=\mathbb{Q} \cap(0,1]$ is dense in $[0,1]$, we have $\int_{0}^{x} \varphi(t) d t=f(x)-f(0)$ for all $x \in[0,1]$. Therefore $\varphi(x)=f^{\prime}(x)$ almost everywhere. This completes the proof.

Added in proof. Professor S. Kanemitsu pointed out that L. J. Mordell ( On the evaluation of some multiple series, J. London Math. Soc. 33 (1958), 368-371) had evaluated the double sum $M(s)=\sum_{m, n>1}(m n(m+n))^{-s}$ for $s=1$ and every even integer $s \geq 2$. The identities (1.2) and (1.4) follow easily from the evaluation of $M(1)$ and $M(2)$, respectively. The author would like to take this opportunity to thank Professor Kanemitsu for this observation.

\section{References}

[1] J. W. L. Glaisher, On the history of Euler's constant, Messenger of Math. (N.S.) 1 (1872), 25-30.

[2] H. Gupta, An identity, Res. Bull. Panjab Univ. (N.S.) 15 (1964), 347-349.

[3] G. H. Hardy and E. M. Wright, An Introduction to the Theory of Numbers, 5th ed., Oxford, 1979.

[4] M. Hata and M. Yamaguti, The Takagi function and its generalization, Japan J. Appl. Math. 1 (1984), 183-199.

[5] J. Lehner and M. New man, Sums involving Farey fractions, Acta Arith. 15 (1969), $181-187$.

DIVISION OF MATHEMATICS

FACULTY OF INTEGRATED HUMAN STUDIES

KYOTO UNIVERSITY

KYOTO 606-01, JAPAN

Received on 15.3.1994

and in revised form on 17.8.1994 